\title{
Fourier Transform for Reactivity Calculation
}

\author{
${ }^{1}$ Daniel Suescun-Diaz, ${ }^{2}$ Jorge H. Figueroa-Jimenez and ${ }^{1}$ Alvaro E. Avendaño-Rodriguez \\ ${ }^{1}$ Departamento de Ciencias Naturales, Facultad de Ciencias Exactas y Naturales, \\ Grupo de Fisica Aplicada FIASUR, Universidad Surcolombiana, \\ Av. Pastrana Borrero-Carrera 1 (CP), 410006 Neiva, Colombia \\ ${ }^{2}$ Departamento de Ciencias Naturales y Matematicas, Facultad de Ingenieria y Ciencias, \\ Grupo de Estadística y Matematica Aplicada, Pontificia Universidad Javeriana Cali, \\ Calle 18 No. 118-250, Cali, Colombia \\ daniel.suescun@usco.edu.co
}

\begin{abstract}
This study presents a new formulation for the calculation of nuclear reactivity using the integral of the inverse point kinetics equation, known as the nuclear power history. The calculation of this integral can be made by approximating the area under the integrand curve through a process of zero-order numerical summation. This method is based on the discrete Fourier transform. This analysis is based on the fact that the reactivity can be written in terms of the summation of a circular convolution with impulse response which is characteristic of linear systems.
\end{abstract}

Key words: Inverse point kinetics equations, reactivity, nuclear power plant, Fourier transform, zero-order numerical summation, linear systems

\section{INTRODUCTION}

In a nuclear power plant, it is crucial to know the value of nuclear reactivity for the safe operation of the nuclear reactor. The measurement of reactivity is essential to the nuclear reactor's control systems, since, it provides information that is important for decision making regarding the control rod motion, borated water or just cooling. The calculation of reactivity is carried out using the inverse point kinetics method which consists of modeling the temporal behavior of the neutron flux. This method is represented by an integral-differential equation in which the integral term conserves the nuclear power history and the differential term is directly related to the reactor's period.

The tasks carried out to calculate the nuclear reactivity are mainly based on the discretization of the term related to the integral of the inverse method equation, known as nuclear power history (Shimazu et al., 1987; Hoogenboom and Sluijs, 1988; Binney and Bakir, 1989; Ansari, 1991; Kitano et al., 2000; Tamura, 2003). A method based on finite differences has also, been presented (Diaz and Martinez, 2010). Another solution is based on the three-and five-point formulas of the Lagrange method (Malmir and Vosoughi, 2013). In another publication (Suescun-Diaz et al., 2016), the discrete version of the Laplace transform was employed to calculate the nuclear reactivity using a Savitzky-Golay filter to reduce fluctuations. In a more recent publication (Suescun-Diaz et al., 2017), a method to calculate the reactivity using the Adams-Bashfort-Moulton Method with a Savitzky-Golay filter to control noise was also presented. This study presents a new formulation for the calculation of reactivity in nuclear reactors using the Fourier transform.

\section{MATERIALS AND METHODS}

Theoretical considerations: The expression for reactivity can be easily obtained from the point kinetics equations (Stacey, 2007). It is a system with seven coupled ordinary differential and non-linear equations which describe the time evolution of both the distribution of neutrons and the concentrations of the delayed neutron precursors in the core of a nuclear reactor:

$$
\begin{gathered}
\frac{d \mathrm{P}(\mathrm{t})}{\mathrm{dt}}=\left[\frac{\rho(\mathrm{t})-\beta}{\Lambda}\right] \mathrm{P}(\mathrm{t})+\sum_{\mathrm{i}=1}^{6} \lambda_{\mathrm{i}} \mathrm{C}_{\mathrm{i}}(\mathrm{t}) \\
\frac{\mathrm{dC}_{\mathrm{i}}(\mathrm{t})}{\mathrm{dt}}=\frac{\beta_{\mathrm{i}}}{\Lambda} \mathrm{P}(\mathrm{t})-\lambda_{\mathrm{i}} \mathrm{C}_{\mathrm{i}}(\mathrm{t}) \quad \mathrm{i}=1,2, \ldots, 6
\end{gathered}
$$

Corresponding Author: Daniel Suescun-Diaz, Departamento de Ciencias Naturales, Facultad de Ciencias Exactas y Naturales, Grupo de Fisica Aplicada FIASUR, Universidad Surcolombiana, Av. Pastrana Borrero-Carrera 1 (CP), 410006 Neiva, Colombia, daniel.suescun@usco.edu.co 
With the following initial conditions:

$$
\begin{gathered}
\mathrm{P}(\mathrm{t}=0)=\mathrm{P}_{0} \\
\mathrm{C}_{\mathrm{i}}(\mathrm{t}=0)=\frac{\beta_{\mathrm{i}}}{\Lambda \lambda_{\mathrm{i}}} \mathrm{P}_{0}
\end{gathered}
$$

Where:

$\mathrm{P}(\mathrm{t})=$ The nuclear power

$\mathrm{C}_{\mathrm{i}}(\mathrm{t})=$ The concentration of the ith group of delayed neutrons precursors

$\rho(\mathrm{t})=$ The reactivity

$\Lambda=$ The prompt neutron generation time

$\beta_{i} \quad=$ The effective fraction of the ith group of delayed neutrons

$\beta=$ The total effective fraction of delayed neutrons $\left.\beta=\sum \beta_{\mathrm{i}}\right)$

$\lambda_{i}=$ The decay constant of the ith group of delayed neutrons precursors

The reactivity resulting from a nuclear Power $\mathrm{P}(\mathrm{t})$ can be determined through an inverse kinetics equation (Duderstadt and Hamilton, 1976):

$$
\begin{aligned}
& \rho(\mathrm{t})=\beta+\frac{\Lambda}{\mathrm{P}(\mathrm{t})} \frac{\mathrm{dP}(\mathrm{t})}{\mathrm{dt}}-\frac{1}{\mathrm{P}(\mathrm{t})} \sum_{\mathrm{i}=1}^{6} \lambda_{\mathrm{i}} \beta_{\mathrm{i}} \\
& {\left[\frac{\left\langle\mathrm{P}_{0}\right\rangle}{\lambda_{\mathrm{i}}} \mathrm{e}^{-\lambda_{\mathrm{i}} \mathrm{t}}+\int_{0}^{\mathrm{t}} \mathrm{e}^{-\lambda_{\mathrm{i}}(\mathrm{t}-\mathrm{t})} \mathrm{P}\left(\mathrm{t}^{\prime}\right) \mathrm{dt}^{\prime}\right]}
\end{aligned}
$$

The integral term in Eq. 5, named nuclear power history can be written as:

$$
\int_{0}^{t} \mathrm{e}^{-\lambda_{i}\left(t-t^{\prime}\right)} P\left(t^{\prime}\right) d t^{\prime}=\int_{0}^{t} h_{i}\left(t-t^{\prime}\right) P\left(t^{\prime}\right) d t^{\prime}=y(t)
$$

The calculation of this integral can be made by approximating the area under the integrand curve through a process of zero-order numerical summation. Assuming that this approximation is reasonable, be $\mathrm{P}[\mathrm{n}], \mathrm{h}_{\mathrm{i}}[\mathrm{n}]$ and $y[n]$ the discrete time functions corresponding to the continuous time functions $\mathrm{P}(\mathrm{t}), \mathrm{h}_{\mathrm{i}}(\mathrm{t})$ and $\mathrm{y}(\mathrm{t})$, respectively. Assuming that $\mathrm{N}$ samples of $\mathrm{P}[\mathrm{n}]$ and $\mathrm{h}_{\mathrm{H}}[\mathrm{n}]$ are used, the differential dt' in Eq. 6 results in T, it is the time step and the function required at the sampled points can be approximated by:

$$
y(t) \approx y[n]=T \sum_{k=0}^{N-1} h_{i}[n-k] P[k]
$$

Equation 7 was obtained by approximating the area under the curve of the integrand in Eq. 5 though a zero-order numerical summation process. A fundamental operation in linear digital signal processing is convolution (Haykin and Veen, 1999). The Discrete Fourier Transform (DFT) is closely connected to Eq. 7. In this case, h[n], P[n] and $y[n]$ are sequences of the same length $N$. Both $P[n]$ and $\mathrm{h}[\mathrm{n}]$ are assumed to be periodically extended outside the range 0 to $\mathrm{N}-1$ which results in $\mathrm{y}[\mathrm{n}]$ being periodic as well. The convolution property of the DFT is given by the following relation:

$$
\operatorname{DFT}\{\mathrm{y}[\mathrm{n}]\}=\operatorname{DFT}\{\mathrm{x}[\mathrm{n}]\} \operatorname{DFT}\{\mathrm{h}[\mathrm{n}]\}
$$

This states that the DFT of the cyclic convolution of two signals is the product of the individual DFTs of the signals. The DFT converts cyclic convolution into multiplication. This property is used to reduce the amount of arithmetic needed for convolution, the inverse of the DFT is denoted as IDFT and has the property:

$$
y[n]=\operatorname{IDFT}\{\operatorname{DFT}\{x[n]\} \operatorname{DFT}\{h[n]\}\}
$$

And is calculated by:

$$
\operatorname{IDFT}\{\mathrm{X}[\mathrm{k}]\}=\mathrm{x}[\mathrm{n}]=(1 / \mathrm{N}) \sum_{\mathrm{k}=0}^{\mathrm{N}-1} \mathrm{X}[\mathrm{k}] \mathrm{W}^{\mathrm{nk}}
$$

The differences between the DFT and the IDFT are the factor of $1 / \mathrm{N}$ and the negative power in the IDFT. If an algorithm exists for the calculation of the DFT, multiplication of the DFT by $1 / \mathrm{N}$ and reversing the order of the elements give an algorithm for the IDFT. An efficient algorithm used for the DFT and IDFT is called the Fast Fourier Transform, FFT (Tolimieri et al.,1997). Equaling Eq. 7-10 and replacing into Eq. 5 one obtains:

$$
\begin{aligned}
& \rho(t)=\beta+\frac{\Lambda}{P(t)} \frac{d P(t)}{d t}-\frac{\left\langle P_{0}\right\rangle}{P(t)} \sum_{i=1}^{6} \beta_{i} \mathrm{e}^{-\lambda_{i} t}- \\
& \frac{T}{P(t)}\left[\left(\sum_{i=1}^{6} \lambda_{i} \beta_{i} h_{i}(t)\right) \otimes P(t)\right]
\end{aligned}
$$

Where:

$\mathrm{h}_{\mathrm{i}}(\mathrm{t})=\mathrm{e}^{-\mathrm{x}_{\mathrm{i}} \mathrm{t}}=$ The impulse response

$\mathrm{T}=\mathrm{A}$ constant that corresponds to the time step $\otimes \quad=$ The circular convolution

Using the distributive property results only in the solution of one single convolution. Equation 11 does not use a linear convolution which is obtained using the discrete Laplace transform (Suescun-Diaz et al., 2016) but instead a circular convolution that is obtained from the discrete version of the Fourier transform. 
Due to the initial condition imposed in the stable state at $\mathrm{t}=0$, the reactivity is null for $\mathrm{t}=0$. This condition is not met, however, by Eq. 11 and in order to meet this critical order condition in $\mathrm{n}=0, \mathrm{y}$ it must be null in Eq. 7 and so, the following adjustment is made:

$$
\bar{y}[n]=y[n]-\frac{1}{2}[h[n] P[0]+h[0] P[n]]
$$

The method represented by Eq. 11 allows us to calculate the reactivity even when the nuclear power has noise up to a standard deviation of $\sigma$ which can be called resistance to noise. For a standard deviation $\sigma=0.001$ the differential term can cause problems in the calculation of reactivity and also, the term $\mathrm{P}(\mathrm{t})$ in the denominator of Eq. 11 which makes these equations non-linear. In order to overcome this problem, we will consider that the power signal has a variation due to the noise with a Gaussian distribution and standard deviation $\sigma$ around the value for average power (Kitano et al., 2000 ) as:

$$
\overline{\mathrm{P}_{\mathrm{i}}}=\frac{1}{\mathrm{~N}} \sum_{\mathrm{j}=1}^{\mathrm{N}} \mathrm{P}_{\mathrm{j}}
$$

Where:

$\mathrm{P}_{\mathrm{j}}=$ The power signal measured

$\overline{\mathrm{P}_{\mathrm{i}}}=$ The average power

In the following section, the most relevant results carried out by numerical simulation are presented. The method used for reference is given by the analytical solution of Eq. 5, the proposed method using the FFT is given by Eq. 11 .

\section{RESULTS AND DISCUSSION}

Below are some results of numerical experiments performed for different forms of nuclear power. The simulation runs consider parameters such as $\lambda_{\mathrm{i}}$ the decay constant of delayed neutrons and $\beta_{1}$ the fraction of delayed neutrons. Typical coefficients for the precursors are given in Table 1 . The generation time is $\Lambda=2 \times 10^{-5}$. In the performed numerical experiments, the exact value of reactivity can be calculated using Eq. 5 which is taken as the reference method. It should be noted that during the simulations, a pseudo-random number generator with a normal distribution and a seed was used using the MATLAB command randn ('state', 200) to allow the reader to reproduce some results that are obtained in this study. Furthermore, we suppose that the noise has a Gaussian distribution with a standard deviation $\sigma$ around
Table 1: Typical precursor coefficients

\begin{tabular}{lllllll}
\hline Groups & 1 & 2 & 3 & 4 & 5 & 6 \\
\hline$\lambda_{\mathrm{i}}\left[\mathrm{s}^{-1}\right]$ & 0.012700 & 0.031700 & 0.115000 & 0.311000 & 1.400000 & 3.870000 \\
$\beta_{\mathrm{i}}$ & 0.000266 & 0.001491 & 0.001316 & 0.002849 & 0.000896 & 0.000182 \\
$\beta_{\mathrm{i} / \beta} / 3$ & 0.038000 & 0.213000 & 0.188000 & 0.407000 & 0.128000 & 0.026000 \\
\hline
\end{tabular}

\begin{tabular}{|c|c|c|}
\hline$\underline{h(\mathrm{sec})}$ & FFT & FFT with fitting \\
\hline 0.001 & 0.30 & $4.01 * 10^{5}$ \\
\hline 0.010 & 3.05 & $4.01 * 10^{3}$ \\
\hline 0.100 & 30.47 & $4.00^{*} 10^{-1}$ \\
\hline 0.500 & 152.34 & 9.66 \\
\hline 1.000 & 304.68 & 35.37 \\
\hline
\end{tabular}

Table 2: Maximum difference in $\mathrm{pcm}$ for nuclear power $\mathrm{P}(\mathrm{t})=\exp$

Table 3: Maximum difference in $\mathrm{pcm}$ for nuclear power $\mathrm{P}(\mathrm{t})=\exp$ \begin{tabular}{lll}
$\left(0.006881^{*} \mathrm{t}\right)$ & \\
\hline $\mathrm{h}(\mathrm{sec})$ & $\mathrm{FFT}$ & FFT with fitting
\end{tabular}

\begin{tabular}{lll}
\hline 0.001 & 0.30 & $4.00 * 10^{-5}$
\end{tabular}

$\begin{array}{lll}0.010 & 3.05 & 4.00 * 10^{-3}\end{array}$

$\begin{array}{lll}0.100 & 30.47 & 4.00^{*} 10^{-1}\end{array}$

$\begin{array}{lll}0.500 & 152.34 & 9.64\end{array}$

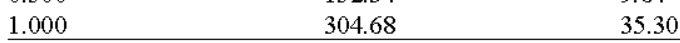

the value for average power provided by Eq. 13. The results obtained in this study coincide well with those reported in a recent publication (Suescun-Diaz et al., 2016).

The first numerical experiment consists in guessing a given form for the nuclear power term as $\mathrm{P}(\mathrm{t})=\exp (\omega \mathrm{t})$ with $\omega=0.12353 ; \omega=0.01046 ; \omega=0.006881$ where, $\omega$ is the positive root value of the inhour equation. These nuclear power variations imply reactivity values $\rho$ of 300 , 70 and $50 \mathrm{pcm}$ for simulation times of 100,300 and $500 \mathrm{sec}$, respectively. Table 2 and 3 show the maximum difference for the FFT with fitting given by Eq. 12 which is the critical condition for the reactor and FFT without fitting.

Practically, not much variation is seen in the results presented and it can be verified that for reactivities between 50 and $300 \mathrm{pcm}$ the method can be used with different time steps (h). Particularly when $h=0.1 \mathrm{sec}$ the maximum difference found is $0.4 \mathrm{pcm}$ which is a very low quantity. This means that this method can be implemented in a digital reactivity meter.

For variations in the nuclear power that produce lower reactivities, it can be seen in Fig. 1 that for a nuclear power $\mathrm{P}(\mathrm{t})=\alpha+\mathrm{b}^{*} \mathrm{t}^{3}$ with $\mathrm{a}=1, \mathrm{~b}=3.67 * 10^{-11}$ and $\mathrm{h}=0.01$ $\mathrm{sec}$, the maximum difference in reactivity is of $0.051 \mathrm{pcm}$ in a time of $\mathrm{t}=2409 \mathrm{sec}$ in relation to the reference value which is a very small difference.

Figure 2 shows the reactivity due to a nuclear power of the form with $b=0.00127$ and time step $h=0.1 \mathrm{sec}$. The reference values were obtained by analytically calculating the expression provided by Eq. 5 and by the method proposed using the FFT set by Eq. 9 and 11. A maximum difference of $0.40 \mathrm{pcm}$ is observed for a simulation time of $\mathrm{t}=10000 \mathrm{sec}$. In relation to the reference 


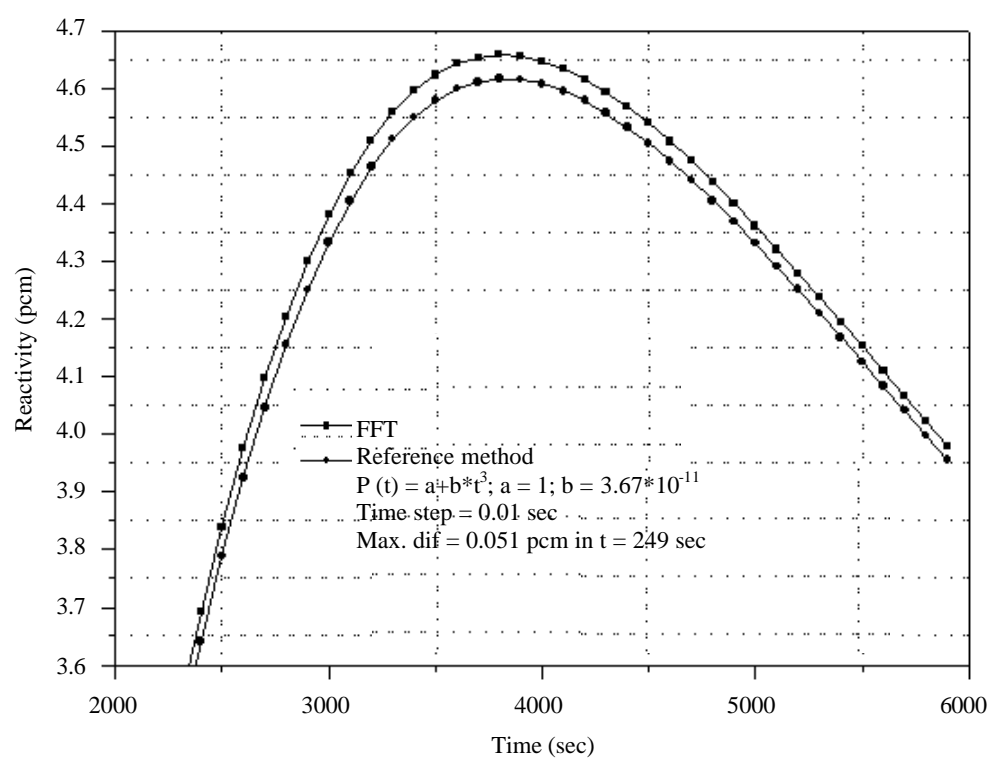

Fig. 1: Variation in nuclear reactivity to produce a power form of $\mathrm{P}(\mathrm{t})=\mathrm{a}+\mathrm{b}^{*} \mathrm{t}^{3}$ with $\mathrm{a}=1$ and $\mathrm{b}=3.67^{*} 10^{-11}$

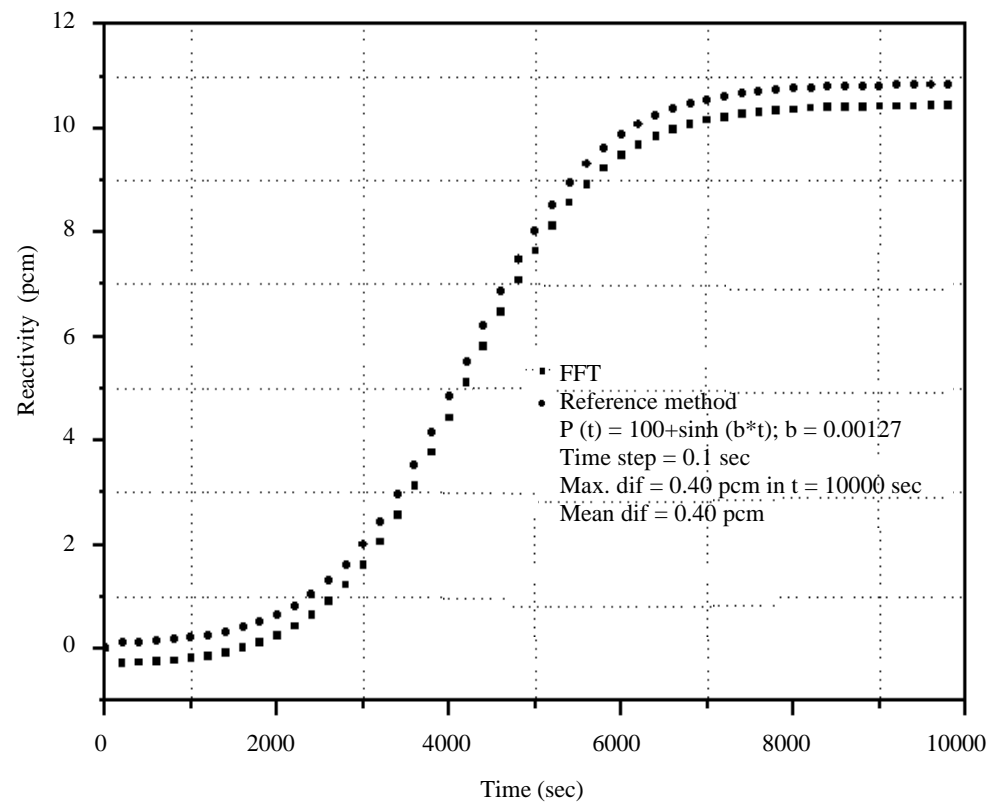

Fig. 2: Variation in nuclear reactivity to produce a power form of $\mathrm{P}(\mathrm{t})=100+\operatorname{Sinh}(\mathrm{bt})$ with $\mathrm{b}=0.00127$

value, it is observed that the method proposed in this study produces an average difference of 0.40 pcm.

The last numerical experiment consists of simulating a more realistic case which is that of a nuclear reactor with noise due to the use of sensors for the detection of the nuclear power which can vary by the introduction of the control rods or the passage of the refrigerant which is either water or water with boron. For a reactivity due to a nuclear power $\mathrm{P}(\mathrm{t})=100+\operatorname{Sinh}(\mathrm{bt})$ with $\mathrm{b}=0.00127$, time step $h=0.1 \mathrm{sec}$, standard deviation $\sigma=0.001$ around the average power and a sample size of $\mathrm{N}=100$, a delay of up to $10 \mathrm{sec}$ could result in the calculation of the value of reactivity. A maximum difference of $3.83 \mathrm{pcm}$ with respect to the reference method for a time $\mathrm{t}=3276 \mathrm{sec}$ is obtained. This shows that the FFT method can be applied to the calculation of reactivity. In this case, it can be said that the method "resists noise" of up to $\sigma=0.001$ (Fig. 3). 


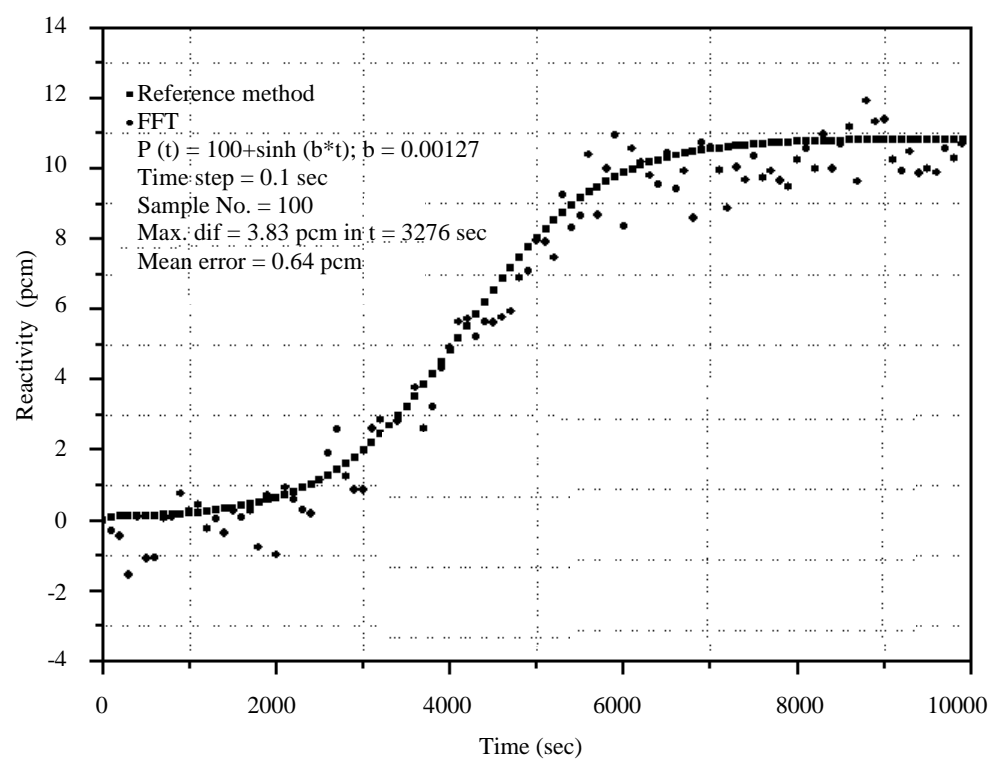

Fig. 3: Variation in nuclear reactivity to produce a power form of $\mathrm{P}(\mathrm{t}) 100+\operatorname{Sinh}(\mathrm{bt})$ with $\mathrm{b}=0.00127$ using standard deviation $\sigma=0.001$

\section{CONCLUSION}

A simple formulation of reactivity was derived by using the inverse point kinetics equation. The implementation is given in a non-recursive form, the non-linear terms in the calculation of reactivity were treated by using the Fourier transform. The simulations show that it is advisable to use a smaller number of samples which would also cause a smaller delay in the calculation of reactivity. The FFT may be used in the calculation of reactivity in nuclear reactors as shown in this study when the nuclear power contains noise with a Gaussian distribution with a standard deviation of up to $\sigma=0.001$ around the average value of the nuclear power with a time step of up to $\Delta \mathrm{t}=0.1 \mathrm{sec}$.

\section{ACKNOWLEDGEMENTS}

This research was carried out thanks to the academic and financial support from the Computational Physics Research Group, the Applied Physics research group (FIASUR) and the Physics Department at the Surcolombian University, Neiva, Colombia.

\section{REFERENCES}

Ansari, S.A., 1991. Development of on-line reactivity meter for nuclear reactors. IEEE. Trans. Nucl. Sci., 38: 946-952.
Binney, S.E. and A.J.M. Bakir, 1989. Design and development of a personal-computer-based reactivity meter for a research reactor. Nucl. Technol., 85: 12-21.

Diaz, D.S. and A.S. Martinez, 2010. Finite differences with exponential filtering in the calculation of reactivity. Kerntechnik, 75: 210-213.

Duderstadt, J.J. and L.J. Hamilton, 1976. Nuclear Reactor Analysis. 2nd Edn., John Wiley \& Sons Inc, New York, USA.,.

Haykin, S. and B.V. Veen, 1999. Signals and Systems. 1st Edn., Wiley, New York, USA., ISBN-13: 978-0471138204, Pages: 720.

Hoogenboom, J.E. and A.R.V.D. Sluijs, 1988. Neutron source strength determination for on-line reactivity measurements. Ann. Nucl. Energy, 15: 553-559.

Kitano, A., M. Itagaki and M. Narita, 2000. Memorial-index-based inverse Kinetics method for continuous measurement of reactivity and source strength. J. Nucl. Sci. Technol., 37: 53-59.

Malmir, H. and N. Vosoughi, 2013. On-line reactivity calculation using Lagrange method. Ann. Nucl. Energy, 62: 463-467.

Shimazu, Y., Y. Nakano, Y. Tahara and T. Okayama, 1987. Development of a compact digital reactivity meter and a reactor physics data processor. Nucl. Technol., 77: $247-254$.

Stacey, W.M., 2007. Nuclear Reactor Physics. 2nd Edn., Wiley-VCH, Weinheim, Germany, ISBN:978-3-527-40679-1, Pages: 706. 
Suescun-Diaz, D., D.R. Causil and J.H. Figueroa-Jimenez, 2017. Adams-Bashforth-Moulton method with Savitzky-Golay filter to reduce reactivity fluctuations. Kerntechnik, 82: 674-677.

Suescun-Diaz, D., H.F. Bonilla-Londono and J.H. Figueroa-Jimenez, 2016. Savitzky-Golay filter for reactivity calculation. J. Nucl. Sci. Technol., 53: 944-950.
Tamura, S., 2003. Signal fluctuation and neutron source in inverse Kinetics method for reactivity measurement in the sub-critical domain. J. Nucl. Sci. Technol., 40: 153-157.

Tolimieri, R., M. An and C. Lu, 1997. Algorithms for Discrete Fourier Transform and Convolution. 2nd Edn., Springer, New York, USA., ISBN: 9780387982618, Pages: 268. 